\title{
Hermite-Hadamard-Fejér Inequality Related to Generalized Convex Functions via Fractional Integrals
}

\author{
M. Rostamian Delavar $\left(\mathbb{D},{ }^{1}\right.$ S. Mohammadi Aslani, ${ }^{2}$ and M. De La Sen $\mathbb{D}^{3}$ \\ ${ }^{1}$ Department of Mathematics, Faculty of Basic Sciences, University of Bojnord, Bojnord, Iran \\ ${ }^{2}$ Department of Mathematics, Karaj Branch, Islamic Azad University, Karaj, Iran \\ ${ }^{3}$ Institute of Research and Development of Processes, University of Basque Country, Campus of Leioa, Aptdo. 644, 48080 Bilbao, Spain
}

Correspondence should be addressed to M. Rostamian Delavar; m.rostamian@ub.ac.ir

Received 16 May 2018; Accepted 26 June 2018; Published 1 August 2018

Academic Editor: S. K. Q. Al-Omari

Copyright (C) 2018 M. Rostamian Delavar et al. This is an open access article distributed under the Creative Commons Attribution License, which permits unrestricted use, distribution, and reproduction in any medium, provided the original work is properly cited.

This paper deals with Hermite-Hadamard-Fejér inequality for $\left(\eta_{1}, \eta_{2}\right)$-convex functions via fractional integrals. Some mid-point and trapezoid type inequalities related to Hermite-Hadamard inequality when the absolute value of derivative of considered function is $\left(\eta_{1}, \eta_{2}\right)$-convex functions are obtained. Furthermore, a refinement for classic Hermite-Hadamard inequality via fractional integrals is given when a positive $\left(\eta_{1}, \eta_{2}\right)$-convex function is increasing.

\section{Introduction and Preliminaries}

In 1906, L. Fejér [1] proved the following integral inequalities which are known in the literature as Fejér inequality:

$$
\begin{gathered}
f\left(\frac{a+b}{2}\right) \int_{a}^{b} g(x) d x \leq \int_{a}^{b} f(x) g(x) d x \\
\quad \leq \frac{f(a)+f(b)}{2} \int_{a}^{b} g(x) d x,
\end{gathered}
$$

where $f:[a ; b] \longrightarrow \mathbb{R}$ is convex and $g:[a, b] \longrightarrow[0, \infty)=$ $\mathbb{R}^{+}$is integrable and symmetric to $x=((a+b) / 2)(g(x)=$ $g(a+b-x), \forall x \in[a, b])$.

If we consider in (1) that $g \equiv 1$, then we recapture the known Hermite-Hadamard inequality.

$$
f\left(\frac{a+b}{2}\right) \leq \frac{1}{b-a} \int_{a}^{b} f(x) d x \leq \frac{f(a)+f(b)}{2} .
$$

On the other hand, the concept of $\left(\eta_{1}, \eta_{2}\right)$-convex has been introduced in $[2,3]$ as a generalization of preinvex functions [4-6] and $\eta$-convex functions [7-9]. We remind these introductory concepts in the following with some geometric interpretation.

\section{(Preinvex Functions)}

Definition 1. A set $I \subseteq \mathbb{R}$ is invex with respect to a real bifunction $\eta: I \times I \longrightarrow \mathbb{R}$, if

$$
x, y \in I, \lambda \in[0,1] \Longrightarrow y+\lambda \eta(x, y) \in I .
$$

If $I$ is an invex set with respect to $\eta$, then a function $f: I \longrightarrow$ $\mathbb{R}$ is said to be preinvex if $x, y \in I$ and $\lambda \in[0,1]$ implies

$$
f(y+\lambda \eta(x, y)) \leq \lambda f(x)+(1-\lambda) f(y) .
$$

In fact, in an invex set for any $x, y \in I$, there is a path starting from $y$ to $y+\eta(x, y)$ which is contained in $I$. The point $x$ is not necessarily the end point of the path. If for every $x, y \in I$ we need that $x$ should be an end point of the path, then $I$ reduces to a convex set.

( $\eta$-Convex Functions)

Definition 2. Consider a convex set $I \subseteq \mathbb{R}$ and a bifunction $\eta: f(I) \times f(I) \longrightarrow \mathbb{R}$. A function $f: I \longrightarrow \mathbb{R}$ is called convex with respect to $\eta$ (briefly $\eta$-convex), if

$$
f(\lambda x+(1-\lambda) y) \leq f(y)+\lambda \eta(f(x), f(y)),
$$

for all $x, y \in I$ and $\lambda \in[0,1]$. 
Geometrically, it says that if a function is $\eta$-convex on $I$, then, for any $x, y \in I$, its graph is on or under the path starting from $(y, f(y))$ and ending at $(x, f(y)+\eta(f(x), f(y)))$. If $f(x)$ should be the end point of the path for every $x, y \in I$, then we have $\eta(x, y)=x-y$ and the function reduces to a convex one.

\section{$\left(\left(\eta_{1}, \eta_{2}\right)\right.$-Convex Functions $)$}

Definition 3. Let $I \subseteq \mathbb{R}$ be an invex set with respect to $\eta_{1}$ : $I \times I \longrightarrow \mathbb{R}$. Consider $f: I \longrightarrow \mathbb{R}$ and $\eta_{2}: f(I) \times f(I) \longrightarrow \mathbb{R}$. The function $f$ is said to be $\left(\eta_{1}, \eta_{2}\right)$-convex if

$$
f\left(x+\lambda \eta_{1}(y, x)\right) \leq f(x)+\lambda \eta_{2}(f(y), f(x)),
$$

for all $x, y \in I$ and $\lambda \in[0,1]$.

Remark 4. An $\left(\eta_{1}, \eta_{2}\right)$-convex function reduces to

(i) a $\eta$-convex function if we consider $\eta_{1}(x, y)=x-y$ for all $x, y \in I$,

(ii) a preinvex function if we consider $\eta_{2}(x, y)=x-y$ for all $x, y \in f(I)$

(iii) a convex function if satisfying (i) and (ii).

Example 5 (see [2]). Consider the function $f: \mathbb{R}^{+} \longrightarrow \mathbb{R}^{+}$by

$$
f(x)= \begin{cases}x, & 0 \leq x \leq 1 \\ 1, & x>1\end{cases}
$$

Define two bifunctions $\eta_{1}: \mathbb{R}^{+} \times \mathbb{R}^{+} \longrightarrow \mathbb{R}$ and $\eta_{2}: \mathbb{R}^{+} \times$ $\mathbb{R}^{+} \longrightarrow \mathbb{R}^{+}$by

$$
\eta_{1}(x, y)= \begin{cases}-y, & 0 \leq y \leq 1 \\ x+y, & y>1\end{cases}
$$

and

$$
\eta_{2}(x, y)= \begin{cases}x+y, & x \leq y \\ 2(x+y), & x>y\end{cases}
$$

Then $f$ is an $\left(\eta_{1}, \eta_{2}\right)$-convex function. But $f$ is not preinvex with respect to $\eta_{1}$ and it is not convex (consider $x=0, y=2$, and $\lambda>0)$.

Also we need the following short preliminaries about the fractional calculus theory which are used throughout the paper.

Definition 6 (see [10]). Consider $f \in L^{1}[a, b]$. The RiemannLiouville integrals $J_{a^{+}}^{\alpha} f$ and $J_{b^{-}}^{\alpha} f$ of order $\alpha>0$ with $a \geq 0$ are defined by

$$
J_{a^{+}}^{\alpha} f(x)=\frac{1}{\Gamma(\alpha)} \int_{a}^{x}(x-t)^{\alpha-1} f(t) d t, \quad x>a,
$$

and

$$
J_{b^{-}}^{\alpha} f(x)=\frac{1}{\Gamma(\alpha)} \int_{x}^{b}(t-x)^{\alpha-1} f(t) d t, \quad x<b
$$

respectively, where

$$
\Gamma(\alpha)=\int_{0}^{\infty} e^{-t} t^{\alpha-1} d t
$$

is Gamma function and $J_{a^{+}}^{0} f(x)=J_{b^{-}}^{0} f(x)=f(x)$.

Fejér inequality for convex functions related to fractional integrals has been obtained in [11] as the following theorem.

Theorem 7. Let $f:[a, b] \longrightarrow \mathbb{R}$ be convex function with $a<b$ and $f \in L^{1}[a, b]$. If $g:[a, b] \longrightarrow \mathbb{R}$ is nonnegative, integrable, and symmetric to $(a+b) / 2$, then the following inequalities hold for fractional integrals:

$$
\begin{aligned}
& f\left(\frac{a+b}{2}\right)\left[J_{a^{+}}^{\alpha} g(b)+J_{b^{-}}^{\alpha} g(a)\right] \\
& \quad \leq\left[J_{a^{+}}^{\alpha}(f g)(b)+J_{b^{-}}^{\alpha}(f g)(a)\right] \\
& \quad \leq \frac{f(a)+f(b)}{2}\left[J_{a^{+}}^{\alpha} g(b)+J_{b^{-}}^{\alpha} g(a)\right],
\end{aligned}
$$

with $\alpha>0$.

Motivated by above works and results, in this paper, we obtain Fejér inequality for $\left(\eta_{1}, \eta_{2}\right)$-convex functions via fractional integrals. Also we give some mid-point and trapezoidtype inequalities related to Hermite-Hadamard inequality when the absolute value of the derivative of the considered function is $\left(\eta_{1}, \eta_{2}\right)$-convex functions. Furthermore, we prove that when a positive $\left(\eta_{1}, \eta_{2}\right)$-convex function is increasing, there exists a refinement for classic Hermite-Hadamard inequality via fractional integrals.

\section{Fejér Inequality}

In this section, we obtain $\left(\eta_{1}, \eta_{2}\right)$-convex version of the Fejér inequality related to fractional integrals. For convenience, we separate this inequality to the left and right.

Theorem 8 (Fejér's left inequality). Let $I \subseteq \mathbb{R}$ be an invex set with respect to $\eta_{1}$ such that

$$
\begin{aligned}
\eta_{1} & \left(x_{2}+t_{2} \eta_{1}\left(x_{1}, x_{2}\right), x_{2}+t_{1} \eta_{1}\left(x_{1}, x_{2}\right)\right) \\
& =\left(t_{2}-t_{1}\right) \eta_{1}\left(x_{1}, x_{2}\right)
\end{aligned}
$$

for all $x_{1}, x_{2} \in I$ and $t_{1}, t_{2} \in[0,1]$. Also let $f: I \longrightarrow \mathbb{R}$ be an $\left(\eta_{1}, \eta_{2}\right)$-convex function, where $\eta_{2}$ is an integrable bifunction on $f(I) \times f(I)$. For any $a, b \in I$ with $\eta_{1}(b, a)>0$, suppose that $f \in L^{1}\left[a, a+\eta_{1}(b, a)\right]$ and the function $g:\left[a, a+\eta_{1}(b, a)\right] \longrightarrow$ $\mathbb{R}^{+}$is integrable and symmetric to $a+(1 / 2) \eta_{1}(b, a)$. Then, for $\alpha>0$, the following inequality holds:

$$
\begin{aligned}
& f\left(\frac{2 a+\eta_{1}(b, a)}{2}\right)\left[J_{a+}^{\alpha} g\left(a+\eta_{1}(b, a)\right)\right. \\
& \left.\quad+J_{\left(a+\eta_{1}(b, a)\right)^{-}}^{\alpha} g(a)\right]-\frac{1}{2 \Gamma(\alpha)}
\end{aligned}
$$




$$
\begin{aligned}
& \cdot \int_{a}^{a+\eta_{1}(b, a)}\left[(x-a)^{\alpha-1}+\left(a+\eta_{1}(b, a)-x\right)^{\alpha-1}\right] \\
& \cdot \eta_{2}\left(f(x), f\left(2 a+\eta_{1}(b, a)-x\right)\right) g(x) d x \\
& \leq\left[J_{a+}^{\alpha}(f g)\left(a+\eta_{1}(b, a)\right)+J_{\left(a+\eta_{1}(b, a)\right)^{-}}^{\alpha}(f g)(a)\right] .
\end{aligned}
$$

Proof. Using condition (14) and the $\left(\eta_{1}, \eta_{2}\right)$-convexity of $f$, we have

$$
\begin{aligned}
& f\left(\frac{2 a+\eta_{1}(b, a)}{2}\right)=f\left(\frac{2 a+(1+t) \eta_{1}(b, a)}{2}-\frac{t}{2}\right. \\
& \left.\cdot \eta_{1}(b, a)\right)=f\left(\frac{2 a+(1+t) \eta_{1}(b, a)}{2}+\frac{1}{2} \eta_{1}(a\right. \\
& \left.\left.\quad+\frac{(1-t)}{2} \eta_{1}(b, a), a+\frac{(1+t)}{2} \eta_{1}(b, a)\right)\right) \\
& \quad=f\left(\frac{2 a+(1+t) \eta_{1}(b, a)}{2}+\frac{1}{2}\right. \\
& \cdot \eta_{1}\left(\frac{2 a+\eta_{1}(b, a)-t \eta_{1}(b, a)}{2},\right. \\
& \left.\left.\quad \frac{2 a+\eta_{1}(b, a)+t \eta_{1}(b, a)}{2}\right)\right) \\
& \quad \leq f\left(\frac{2 a+(1+t) \eta_{1}(b, a)}{2}\right)+\frac{1}{2} \\
& \quad \eta_{2}\left(f\left(\frac{2 a+(1-t) \eta_{1}(b, a)}{2}\right)\right. \\
& \left.f\left(\frac{2 a+(1+t) \eta_{1}(b, a)}{2}\right)\right),
\end{aligned}
$$

and with the same argument as above we have

$$
\begin{aligned}
& f\left(\frac{2 a+\eta_{1}(b, a)}{2}\right) \leq f\left(\frac{2 a+(1-t) \eta_{1}(b, a)}{2}\right)+\frac{1}{2} \\
& \cdot \eta_{2}\left(f\left(\frac{2 a+(1+t) \eta_{1}(b, a)}{2}\right),\right. \\
& \left.f\left(\frac{2 a+(1-t) \eta_{1}(b, a)}{2}\right)\right) .
\end{aligned}
$$

By the use of Definition 6 and two changes of variable

$$
x=\frac{2 a+(1-t) \eta_{1}(b, a)}{2}
$$

and

$$
x=\frac{2 a+(1+t) \eta_{1}(b, a)}{2}
$$

in (16) and (17), respectively, we obtain the following inequalities:

$$
\begin{aligned}
K_{1} & =\Gamma(\alpha) J_{\left(a+\eta_{1}(b, a)\right)^{-}}^{\alpha} f(a) g(a)=\int_{a}^{a+\eta_{1}(b, a)}(x \\
& -a)^{\alpha-1} f(x) g(x) d x=\int_{a}^{a+(1 / 2) \eta_{1}(b, a)}(x-a)^{\alpha-1}
\end{aligned}
$$$$
\cdot f(x) g(x) d x+\int_{a+(1 / 2) \eta_{1}(b, a)}^{a+\eta_{1}(b, a)}(x-a)^{\alpha-1} f(x)
$$$$
g(x) d x=\left(\frac{\eta_{1}(b, a)}{2}\right)^{\alpha}\left[\int_{0}^{1}(1-t)^{\alpha-1}\right.
$$$$
\cdot f\left(\frac{2 a+(1-t) \eta_{1}(b, a)}{2}\right)
$$$$
\cdot g\left(\frac{2 a+(1-t) \eta_{1}(b, a)}{2}\right) d t+\int_{0}^{1}(1+t)^{\alpha-1}
$$$$
f\left(\frac{2 a+(1+t) \eta_{1}(b, a)}{2}\right)
$$$$
\left.\cdot g\left(\frac{2 a+(1+t) \eta_{1}(b, a)}{2}\right) d t\right]
$$$$
\geq\left(\frac{\eta_{1}(b, a)}{2}\right)^{\alpha}\left[\int_{0}^{1}(1-t)^{\alpha-1} f\left(\frac{2 a+\eta_{1}(b, a)}{2}\right)\right.
$$$$
g\left(\frac{2 a+(1-t) \eta_{1}(b, a)}{2}\right) d t-\frac{1}{2} \int_{0}^{1}(1
$$

$$
-t)^{\alpha-1} \eta_{2}\left(f\left(\frac{2 a+(1+t) \eta_{1}(b, a)}{2}\right),\right.
$$$$
\left.f\left(\frac{2 a+(1-t) \eta_{1}(b, a)}{2}\right)\right)
$$$$
\times g\left(\frac{2 a+(1-t) \eta_{1}(b, a)}{2}\right) d t+\int_{0}^{1}(1+t)^{\alpha-1}
$$$$
f\left(\frac{2 a+\eta_{1}(b, a)}{2}\right)
$$$$
g\left(\frac{2 a+(1+t) \eta_{1}(b, a)}{2}\right) d t-\frac{1}{2} \int_{0}^{1}(1
$$$$
+t)^{\alpha-1} \eta_{2}\left(f\left(\frac{2 a+(1-t) \eta_{1}(b, a)}{2}\right),\right.
$$$$
\left.f\left(\frac{2 a+(1+t) \eta_{1}(b, a)}{2}\right)\right)
$$$$
\left.\times g\left(\frac{2 a+(1+t) \eta_{1}(b, a)}{2}\right) d t\right] .
$$

The simple form of (20) along with the fact that $g$ is symmetric to $a+(1 / 2) \eta_{1}(b, a)$ leads to the following relations:

$$
K_{1} \geq f\left(\frac{2 a+\eta_{1}(b, a)}{2}\right) \int_{a}^{a+(1 / 2) \eta_{1}(b, a)}(x-a)^{\alpha-1}
$$




$$
\begin{aligned}
& g(x) d x-\frac{1}{2} \\
& \int_{a}^{a+(1 / 2) \eta_{1}(b, a)}\left(a+\eta_{1}(b, a)-x\right)^{\alpha-1} \\
& \cdot \eta_{2}\left(f(x), f\left(2 a+\eta_{1}(b, a)-x\right)\right) g(x) d x \\
& +f\left(\frac{2 a+\eta_{1}(b, a)}{2}\right) \int_{a+(1 / 2) \eta_{1}(b, a)}^{a+\eta_{1}(b, a)}(x-a)^{\alpha-1} \\
& g(x) d x-\frac{1}{2} \int_{a+(1 / 2) \eta_{1}(b, a)}^{a+\eta_{1}(b, a)}\left(a+\eta_{1}(b, a)-x\right)^{\alpha-1} \\
& \text { - } \eta_{2}\left(f(x), f\left(2 a+\eta_{1}(b, a)-x\right)\right) g(x) d x \\
& =f\left(\frac{2 a+\eta_{1}(b, a)}{2}\right) \int_{a}^{a+\eta_{1}(b, a)}(x-a)^{\alpha-1} g(x) d x \\
& -\frac{1}{2} \int_{a}^{a+\eta_{1}(b, a)}\left(a+\eta_{1}(b, a)-x\right)^{\alpha-1} \\
& \text { - } \eta_{2}\left(f(x), f\left(2 a+\eta_{1}(b, a)-x\right)\right) g(x) d x \\
& =\Gamma(\alpha) f\left(\frac{2 a+\eta_{1}(b, a)}{2}\right) J_{\left(a+\eta_{1}(b, a)\right)^{-}}^{\alpha} g(a)-\frac{1}{2} \\
& \int_{a}^{a+\eta_{1}(b, a)}\left(a+\eta_{1}(b, a)-x\right)^{\alpha-1} \\
& \cdot \eta_{2}\left(f(x), f\left(2 a+\eta_{1}(b, a)-x\right)\right) g(x) d x \text {. }
\end{aligned}
$$

Also with the same argument as above we have

$$
\begin{aligned}
K_{2} & =\Gamma(\alpha) J_{a^{+}}^{\alpha} f\left(a+\eta_{1}(b, a)\right) g\left(a+\eta_{1}(b, a)\right) \\
& =\int_{a}^{a+\eta_{1}(b, a)}\left(a+\eta_{1}(b, a)-x\right)^{\alpha-1} f(x) g(x) d x \\
& \geq \Gamma(\alpha) f\left(\frac{2 a+\eta_{1}(b, a)}{2}\right) J_{a^{+}}^{\alpha} g\left(a+\eta_{1}(b, a)\right)-\frac{1}{2} \\
& \cdot \int_{a}^{a+\eta_{1}(b, a)}(x-a)^{\alpha-1} \\
& \cdot \eta_{2}\left(f(x), f\left(2 a+\eta_{1}(b, a)-x\right)\right) g(x) d x .
\end{aligned}
$$

Now adding $K_{1}$ to $K_{2}$ implies the result.

To obtain the right part of the Fejér inequality related to fractional integrals, we need a primary lemma.

Lemma 9. Let $I \subseteq \mathbb{R}$ be an invex set with respect to $\eta_{1}$ : $I \times I \longrightarrow \mathbb{R}$ and $a, b \in I$ with $\eta_{1}(b, a)>0$. If $g:[a, a+$ $\left.\eta_{1}(b, a)\right] \longrightarrow \mathbb{R}$ is integrable and symmetric to $a+(1 / 2) \eta_{1}(b, a)$, then

$$
\begin{aligned}
J_{a^{+}}^{\alpha} g\left(a+\eta_{1}(b, a)\right)=J_{\left(a+\eta_{1}(b, a)\right)^{-}}^{\alpha} g(a) \\
\quad=\frac{1}{2}\left[J_{a^{+}}^{\alpha} g\left(a+\eta_{1}(b, a)\right)+J_{\left(a+\eta_{1}(b, a)\right)^{-}}^{\alpha} g(a)\right] .
\end{aligned}
$$

Proof. Since $g$ is symmetric to $a+(1 / 2) \eta_{1}(b, a)$, we have $g(2 a+$ $\left.\eta_{1}(b, a)-x\right)=g(x)$ for all $x \in\left[a, a+\eta_{1}(b, a)\right]$. Then

$$
\begin{aligned}
& J_{a^{+}}^{\alpha} g\left(a+\eta_{1}(b, a)\right) \\
& \quad=\frac{1}{\Gamma(\alpha)} \int_{a}^{a+\eta_{1}(b, a)}\left(a+\eta_{1}(b, a)-x\right)^{\alpha-1} g(x) d x \\
& \quad=\frac{1}{\Gamma(\alpha)} \int_{a}^{a+\eta_{1}(b, a)}(t-a)^{\alpha-1} g\left(2 a+\eta_{1}(b, a)-t\right) d t \\
& \quad=\frac{1}{\Gamma(\alpha)} \int_{a}^{a+\eta_{1}(b, a)}(t-a)^{\alpha-1} g(t) d t \\
& \quad=J_{\left(a+\eta_{1}(b, a)\right)^{-}}^{\alpha} g(a) .
\end{aligned}
$$

Theorem 10 (Fejér's right inequality). Let $I \subseteq \mathbb{R}$ be an invex set with respect to $\eta_{1}$ and let $f: I \longrightarrow \mathbb{R}$ be an $\left(\eta_{1}, \eta_{2}\right)$-convex function, where $\eta_{2}$ is an integrable bifunction on $f(I) \times f(I)$. For any $a, b \in I$ with $\eta_{1}(b, a)>0$, suppose that the function $g:\left[a, a+\eta_{1}(b, a)\right] \longrightarrow \mathbb{R}^{+}$is integrable and symmetric to $a+(1 / 2) \eta_{1}(b, a)$ and $f \in L^{1}\left[a, a+\eta_{1}(b, a)\right]$. Then, for $\alpha>0$, the following inequality holds:

$$
\begin{aligned}
& {\left[J_{a+}^{\alpha}(f g)\left(a+\eta_{1}(b, a)\right)+J_{\left(a+\eta_{1}(b, a)\right)^{-}}^{\alpha}(f g)(a)\right]} \\
& \quad \leq\left(\frac{2 f(a)+\eta_{2}(f(b), f(a))}{2}\right) \\
& \quad \cdot\left[J_{a+}^{\alpha} g\left(a+\eta_{1}(b, a)\right)+J_{\left(a+\eta_{1}(b, a)\right)^{-}}^{\alpha} g(a)\right] .
\end{aligned}
$$

Proof. From $\left(\eta_{1}, \eta_{2}\right)$-convexity of $f$, using the changes of variables $x=a+t \eta_{1}(b, a)$ and $x=a+(1-t) \eta_{1}(b, a)$, respectively, we obtain the two following inequalities:

$$
\begin{aligned}
& \frac{\Gamma(\alpha)}{\eta_{1}^{\alpha}(b, a)} J_{\left(a+\eta_{1}(b, a)\right)^{-}}^{\alpha} f(a) g(a)=\frac{1}{\eta_{1}^{\alpha}(b, a)} \\
& \cdot \int_{a}^{a+\eta_{1}(b, a)}(x-a)^{\alpha-1} f(x) g(x) d x \\
& \leq \int_{0}^{1} t^{\alpha-1}\left[f(a)+t \eta_{2}(f(b), f(a))\right] \\
& \cdot g\left(a+t \eta_{1}(b, a)\right) d t=f(a) \\
& \cdot \int_{0}^{1} t^{\alpha-1} g\left(a+t \eta_{1}(b, a)\right) d t+\eta_{2}(f(b), f(a)) \\
& \cdot \int_{0}^{1} t^{\alpha} g\left(a+t \eta_{1}(b, a)\right) d t
\end{aligned}
$$

and

$$
\begin{aligned}
& \frac{\Gamma(\alpha)}{\eta_{1}^{\alpha}(b, a)} J_{a^{+}}^{\alpha} f\left(a+\eta_{1}(b, a)\right) g\left(a+\eta_{1}(b, a)\right) \\
& \quad=\frac{1}{\eta_{1}^{\alpha}(b, a)} \int_{a}^{a+\eta_{1}(b, a)}\left(a+\eta_{1}(b, a)-x\right)^{\alpha-1} f(x)
\end{aligned}
$$




$$
\begin{aligned}
& \cdot g(x) d x \\
& \leq \int_{0}^{1} t^{\alpha-1}\left[f(a)+(1-t) \eta_{2}(f(b), f(a))\right] \\
& \cdot g\left(a+(1-t) \eta_{1}(b, a)\right) d t=f(a) \\
& \cdot \int_{0}^{1} t^{\alpha-1} g\left(a+(1-t) \eta_{1}(b, a)\right) d t \\
& +\eta_{2}(f(b), f(a)) \int_{0}^{1} t^{\alpha-1}(1-t) \\
& \cdot g\left(a+(1-t) \eta_{1}(b, a)\right) d t .
\end{aligned}
$$

Now adding (26) to (27) with the fact that $g$ is symmetric to $a+(1 / 2) \eta_{1}(b, a)$ implies that

$$
\begin{aligned}
& \frac{\Gamma(\alpha)}{\eta_{1}^{\alpha}(b, a)}\left[J_{a^{+}}^{\alpha} f\left(a+\eta_{1}(b, a)\right) g\left(a+\eta_{1}(b, a)\right)\right. \\
& \left.\quad+J_{\left(a+\eta_{1}(b, a)\right)^{-}}^{\alpha} f(a) g(a)\right] \\
& \quad \leq\left[2 f(a) \int_{0}^{1} t^{\alpha-1} g\left(a+t \eta_{1}(b, a)\right) d t\right. \\
& \left.\quad+\eta_{2}(f(b), f(a)) \int_{0}^{1} t^{\alpha-1} g\left(a+t \eta_{1}(b, a)\right) d t\right] \\
& \quad=\left(2 f(a)+\eta_{2}(f(b), f(a))\right) \\
& \quad \cdot \int_{0}^{1} t^{\alpha-1} g\left(a+t \eta_{1}(b, a)\right) d t=\frac{\Gamma(\alpha)}{\eta_{1}^{\alpha}(b, a)}(2 f(a) \\
& \left.\quad+\eta_{2}(f(b), f(a))\right) J_{\left(a+\eta_{1}(b, a)\right)^{-}}^{\alpha} g(a) .
\end{aligned}
$$

Now by the use of Lemma 9 we have

$$
\begin{aligned}
& \frac{\Gamma(\alpha)}{\eta_{1}^{\alpha}(b, a)}\left[J_{a^{+}}^{\alpha} f\left(a+\eta_{1}(b, a)\right) g\left(a+\eta_{1}(b, a)\right)\right. \\
& \left.\quad+J_{\left(a+\eta_{1}(b, a)\right)^{-}}^{\alpha} f(a) g(a)\right] \\
& \quad \leq \frac{\Gamma(\alpha)}{\eta_{1}^{\alpha}(b, a)}\left(\frac{2 f(a)+\eta_{2}(f(b), f(a))}{2}\right) \\
& \cdot\left[J_{a^{+}}^{\alpha} g\left(a+\eta_{1}(b, a)\right)+J_{\left(a+\eta_{1}(b, a)\right)^{-}}^{\alpha} g(a)\right],
\end{aligned}
$$

which implies the respected inequality.

Corollary 11. If in Theorems 8 and 10 we consider

(i) $\alpha=1$, then the following inequality holds, which is the classical form of Fejerr inequality related to $\left(\eta_{1}, \eta_{2}\right)$-convex functions:

$$
\begin{aligned}
& f\left(\frac{2 a+\eta_{1}(b, a)}{2}\right) \int_{a}^{a+\eta_{1}(b, a)} g(x) d x-\frac{1}{2} \\
& \quad \cdot \int_{a}^{a+\eta_{1}(b, a)} \eta_{2}\left(f(x), f\left(2 a+\eta_{1}(b, a)-x\right)\right)
\end{aligned}
$$

$$
\begin{aligned}
& \cdot g(x) d x \leq \int_{a}^{a+\eta_{1}(b, a)} f(x) g(x) d x \leq[f(a) \\
& \left.+\frac{\eta_{2}(f(b), f(a))}{2}\right] \int_{a}^{a+\eta_{1}(b, a)} g(x) d x
\end{aligned}
$$

(ii) $g \equiv 1$, then we get Hermite-Hadamard inequality for $\left(\eta_{1}, \eta_{2}\right)$-convex functions as follows:

$$
\begin{aligned}
f\left(\frac{2 a+\eta_{1}(b, a)}{2}\right) & \leq \frac{\Gamma(\alpha+1)}{2(b-a)^{\alpha}}\left[J_{a^{+}}^{\alpha} f(b)+J_{b^{-}}^{\alpha} f(a)\right] \\
& \leq\left(\frac{2 f(a)+\eta_{2}(f(b), f(a))}{2}\right)
\end{aligned}
$$

which is a generalization of inequality (2.1) in [12].

Corollary 12. If in Theorems 8 and 10 we set $\eta_{1}(x, y)=x-y$ for all $x, y \in I$, then we obtain Fejér inequality for fractional integrals related to $\eta$-convex functions.

$$
\begin{aligned}
& f\left(\frac{a+b}{2}\right)\left[J_{a^{+}}^{\alpha} g(b)+J_{b^{-}}^{\alpha} g(a)\right] \\
& \leq\left[J_{a^{+}}^{\alpha}(f g)(b)+J_{b^{-}}^{\alpha}(f g)(a)\right] \\
& \quad \leq\left(\frac{2 f(a)+\eta_{2}(f(b), f(a))}{2}\right) \\
& \quad \cdot\left[J_{a^{+}}^{\alpha} g(b)+J_{b^{-}}^{\alpha} g(a)\right] .
\end{aligned}
$$

Corollary 13. If in Theorems 8 and 10 we set $\eta_{2}(x, y)=x-y$ for all $x, y \in f(I)$, then we obtain Fejér inequality for fractional integrals related to preinvex functions.

$$
\begin{aligned}
& f\left(\frac{2 a+\eta_{1}(b, a)}{2}\right) \\
& \cdot\left[J_{a^{+}}^{\alpha} g\left(a+\eta_{1}(b, a)\right)+J_{\left(a+\eta_{1}(b, a)\right)^{-}}^{\alpha} g(a)\right] \\
& \leq\left[J_{a^{+}}^{\alpha}(f g)\left(a+\eta_{1}(b, a)\right)+J_{\left(a+\eta_{1}(b, a)\right)^{-}}^{\alpha}(f g)(a)\right] \\
& \leq\left(\frac{f(a)+f(b)}{2}\right) \\
& \quad \cdot\left[J_{a^{+}}^{\alpha} g\left(a+\eta_{1}(b, a)\right)+J_{\left(a+\eta_{1}(b, a)\right)^{-}}^{\alpha} g(a)\right] .
\end{aligned}
$$

Corollary 14 (see [11]). With all conditions of Corollaries 12 and 13, we have the classic Fejér inequality for fractional integrals.

$$
\begin{aligned}
& f\left(\frac{a+b}{2}\right)\left[J_{a^{+}}^{\alpha} g(b)+J_{b^{-}}^{\alpha} g(a)\right] \\
& \quad \leq\left[J_{a^{+}}^{\alpha}(f g)(b)+J_{b^{-}}^{\alpha}(f g)(a)\right] \\
& \quad \leq\left(\frac{f(a)+f(b)}{2}\right)\left[J_{a^{+}}^{\alpha} g(b)+J_{b^{-}}^{\alpha} g(a)\right] .
\end{aligned}
$$


Corollary 15 (see [12]). If in (34) we consider $g \equiv 1$, then we recapture Hermite-Hadamard inequality for fractional integrals in convex case.

$$
\begin{aligned}
f\left(\frac{a+b}{2}\right) & \leq \frac{\Gamma(\alpha+1)}{2(b-a)^{\alpha}}\left[J_{a^{+}}^{\alpha}(f)(b)+J_{b^{-}}^{\alpha}(f)(a)\right] \\
& \leq \frac{f(a)+f(b)}{2} .
\end{aligned}
$$

\section{Mid-Point and Trapezoid-Type Inequalities}

In this section, we obtain, respectively, the mid-point and trapezoid-type inequalities related to (31) when the absolute value of the derivative of the considered function is $\left(\eta_{1}, \eta_{2}\right)$ convex. In fact, by mid-point-type inequality we mean estimating the difference between left and middle parts of (31) and by trapezoid-type inequality we mean estimating the difference between right and middle parts of (31).

The following lemma is generalization of Lemma 1 obtained in [13] to the preinvex case.

Lemma 16. Let $I \subseteq \mathbb{R}$ be an open invex set with respect to $\eta_{1}: I \times I \longrightarrow \mathbb{R}$ and let $f: I \longrightarrow \mathbb{R}$ be a differentiable function. For any $a, b \in I$ with $\eta_{1}(b, a)>0$, if $f \in L^{1}\left[a, a+\eta_{1}(b, a)\right]$, then the following equality for fractional integrals holds:

$$
\begin{aligned}
& f\left(\frac{2 a+\eta_{1}(b, a)}{2}\right) \\
& -\frac{\Gamma(\alpha+1)}{2 \eta_{1}^{\alpha}(b, a)}\left[J_{a^{+}}^{\alpha} f\left(a+\eta_{1}(b, a)\right)+J_{\left(a+\eta_{1}(b, a)\right)^{-}}^{\alpha} f(a)\right] \\
& =\frac{\eta_{1}(b, a)}{2} \sum_{k=1}^{4} I_{k}
\end{aligned}
$$

where

$$
\begin{aligned}
& I_{1}=\int_{0}^{1 / 2} t^{\alpha} f^{\prime}\left(a+t \eta_{1}(b, a)\right) d t, \\
& I_{2}=\int_{0}^{1 / 2}\left(-t^{\alpha}\right) f^{\prime}\left(a+(1-t) \eta_{1}(b, a)\right) d t, \\
& I_{3}=\int_{1 / 2}^{1}\left(t^{\alpha}-1\right) f^{\prime}\left(a+t \eta_{1}(b, a)\right) d t, \\
& I_{4}=\int_{1 / 2}^{1}\left(1-t^{\alpha}\right) f^{\prime}\left(a+(1-t) \eta_{1}(b, a)\right) d t .
\end{aligned}
$$

Proof. Integrating by parts in $I_{1}$ implies that

$$
\begin{aligned}
I_{1} & =\int_{0}^{1 / 2} t^{\alpha} f^{\prime}\left(a+t \eta_{1}(b, a)\right) d t \\
& =\left.\frac{t^{\alpha} f\left(a+t \eta_{1}(b, a)\right)}{\eta_{1}(b, a)}\right|_{0} ^{1 / 2}
\end{aligned}
$$

$$
\begin{aligned}
& -\frac{\alpha}{\eta_{1}(b, a)} \int_{0}^{1 / 2} t^{\alpha-1} f\left(a+t \eta_{1}(b, a)\right) d t \\
= & \frac{2^{-\alpha}}{\eta_{1}(b, a)} f\left(\frac{2 a+\eta_{1}(b, a)}{2}\right) \\
& -\frac{\alpha}{\eta_{1}(b, a)} \int_{0}^{1 / 2} t^{\alpha-1} f\left(a+t \eta_{1}(b, a)\right) d t .
\end{aligned}
$$

Similarly, we have

$$
\begin{aligned}
I_{2}= & \frac{2^{-\alpha}}{\eta_{1}(b, a)} f\left(\frac{2 a+\eta_{1}(b, a)}{2}\right) \\
& -\frac{\alpha}{\eta_{1}(b, a)} \int_{0}^{1 / 2} t^{\alpha-1} f\left(a+(1-t) \eta_{1}(b, a)\right) d t, \\
I_{3}= & \int_{1 / 2}^{1}\left(t^{\alpha}-1\right) f^{\prime}\left(a+t \eta_{1}(b, a)\right) d t \\
= & \left.\frac{\left(t^{\alpha}-1\right) f\left(a+t \eta_{1}(b, a)\right)}{\eta_{1}(b, a)}\right|_{1 / 2} ^{1} \\
& -\frac{\alpha}{\eta_{1}(b, a)} \int_{1 / 2}^{1} t^{\alpha-1} f\left(a+t \eta_{1}(b, a)\right) d t \\
= & \frac{1-2^{-\alpha}}{\eta_{1}(b, a)} f\left(\frac{2 a+\eta_{1}(b, a)}{2}\right) \\
& -\frac{\alpha}{\eta_{1}(b, a)} \int_{1 / 2}^{1} t^{\alpha-1} f\left(a+t \eta_{1}(b, a)\right) d t, \\
I_{4}= & \frac{1-2^{-\alpha}}{\eta_{1}(b, a)} f\left(\frac{2 a+\eta_{1}(b, a)}{2}\right) \\
& -\frac{\alpha}{\eta_{1}(b, a)} \int_{1 / 2}^{1} t^{\alpha-1} f\left(a+(1-t) \eta_{1}(b, a)\right) d t .
\end{aligned}
$$

Now, by adding all of above equalities, we get to the desired result.

$$
\begin{aligned}
I_{1} & +I_{2}+I_{3}+I_{4}=\frac{2}{\eta_{1}(b, a)} f\left(\frac{2 a+\eta_{1}(b, a)}{2}\right) \\
& -\frac{\alpha}{\eta_{1}(b, a)}\left[\int_{0}^{1} t^{\alpha-1} f\left(a+t \eta_{1}(b, a)\right) d t\right. \\
& \left.+\int_{0}^{1} t^{\alpha-1} f\left(a+(1-t) \eta_{1}(b, a)\right) d t\right]=\frac{2}{\eta_{1}(b, a)} \\
& \cdot f\left(\frac{2 a+\eta_{1}(b, a)}{2}\right) \\
& -\frac{\Gamma(\alpha+1)}{\eta_{1}^{\alpha+1}(b, a)}\left[J_{\left(a+\eta_{1}(b, a)\right)^{-}}^{\alpha} f(a)\right. \\
& \left.+J_{a^{+}}^{\alpha} f\left(a+\eta_{1}(b, a)\right)\right] .
\end{aligned}
$$


The mid-point-type inequality related to (31) is obtained in the following.

Theorem 17. Let $I \subseteq \mathbb{R}$ be an open invex set with respect to $\eta_{1}: I \times I \longrightarrow \mathbb{R}$ and let $f: I \longrightarrow \mathbb{R}$ be a differentiable function. Suppose that $\left|f^{\prime}\right|$ is an $\left(\eta_{1}, \eta_{2}\right)$-convex function on $I$ and, for any $a, b \in I$ with $\eta_{1}(b, a)>0, f^{\prime} \in L^{1}\left[a, a+\eta_{1}(b, a)\right]$. Then

$$
\begin{aligned}
& \mid f\left(\frac{2 a+\eta_{1}(b, a)}{2}\right)-\frac{\Gamma(\alpha+1)}{2 \eta_{1}^{\alpha}(b, a)}\left[J_{a^{+}}^{\alpha} f\left(a+\eta_{1}(b, a)\right)\right. \\
& \left.\quad+J_{\left(a+\eta_{1}(b, a)\right)^{-}}^{\alpha} f(a)\right] \mid \leq \frac{\eta_{1}(b, a)}{2^{\alpha+1}(\alpha+1)}\left\{\left|f^{\prime}(a)\right|\right. \\
& \quad+\left|f^{\prime}(b)\right|+\frac{1}{2} \eta_{2}\left(\left|f^{\prime}(a)\right|,\left|f^{\prime}(b)\right|\right)+\frac{1}{2} \\
& \left.\quad \cdot \eta_{2}\left(\left|f^{\prime}(b)\right|,\left|f^{\prime}(a)\right|\right)\right\} \\
& \text { for } 0<\alpha \leq 1 .
\end{aligned}
$$

Proof. Using Lemma 16, we get

$$
\begin{aligned}
& \mid f\left(\frac{2 a+\eta_{1}(b, a)}{2}\right)-\frac{\Gamma(\alpha+1)}{2 \eta_{1}^{\alpha}(b, a)}\left[J_{a^{+}}^{\alpha} f\left(a+\eta_{1}(b, a)\right)\right. \\
& \left.\quad+J_{\left(a+\eta_{1}(b, a)\right)^{-}}^{\alpha} f(a)\right]\left|\leq \frac{\eta_{1}(b, a)}{2} \sum_{k=1}^{4}\right| I_{k} \mid .
\end{aligned}
$$

Now, using $\left(\eta_{1}, \eta_{2}\right)$-convexity of $\left|f^{\prime}\right|$, we obtain

$$
\begin{aligned}
\left|I_{1}\right| \leq & \int_{0}^{1 / 2} t^{\alpha}\left|f^{\prime}\left(a+t \eta_{1}(b, a)\right)\right| d t \\
\leq & \int_{0}^{1 / 2} t^{\alpha}\left|f^{\prime}(a)\right| d t \\
& +\int_{0}^{1 / 2} t^{\alpha+1} \eta_{2}\left(\left|f^{\prime}(b)\right|,\left|f^{\prime}(a)\right|\right) d t \\
= & \frac{1}{2^{\alpha+1}(\alpha+1)}\left|f^{\prime}(a)\right| \\
& +\frac{1}{2^{\alpha+2}(\alpha+2)} \eta_{2}\left(\left|f^{\prime}(b)\right|,\left|f^{\prime}(a)\right|\right) .
\end{aligned}
$$

Analogously,

$$
\begin{aligned}
\left|I_{2}\right| \leq & \frac{1}{2^{\alpha+1}(\alpha+1)}\left|f^{\prime}(b)\right| \\
& +\frac{1}{2^{\alpha+2}(\alpha+2)} \eta_{2}\left(\left|f^{\prime}(a)\right|,\left|f^{\prime}(b)\right|\right) .
\end{aligned}
$$

Also, using the fact that

$$
\left|t_{1}^{\alpha}-t_{2}^{\alpha}\right| \leq\left|t_{1}-t_{2}\right|^{\alpha}
$$

for all $\alpha \in(0,1]$ and $t_{1}, t_{2} \in[0,1]$, we have

$$
\begin{aligned}
\left|I_{3}\right| \leq & \frac{1}{2^{\alpha+1}(\alpha+1)}\left|f^{\prime}(a)\right| \\
& +\frac{\alpha+3}{2^{\alpha+2}(\alpha+1)(\alpha+2)} \eta_{2}\left(\left|f^{\prime}(b)\right|,\left|f^{\prime}(a)\right|\right),
\end{aligned}
$$

and

$$
\begin{aligned}
\left|I_{4}\right| \leq & \frac{1}{2^{\alpha+1}(\alpha+1)}\left|f^{\prime}(b)\right| \\
& +\frac{\alpha+3}{2^{\alpha+2}(\alpha+1)(\alpha+2)} \eta_{2}\left(\left|f^{\prime}(a)\right|,\left|f^{\prime}(b)\right|\right) .
\end{aligned}
$$

Now adding all of above inequalities implies the required result.

Corollary 18. If in Theorem 17 we consider $\eta_{1}(x, y)=x-y$ for all $x, y \in I$, then

$$
\begin{aligned}
& \left|f\left(\frac{a+b}{2}\right)-\frac{\Gamma(\alpha+1)}{2(b-a)^{\alpha}}\left[J_{a^{+}}^{\alpha} f(b)+J_{(b)^{-}}^{\alpha} f(a)\right]\right| \\
& \quad \leq \frac{(b-a)}{2^{\alpha+1}(\alpha+1)}\left\{\left|f^{\prime}(a)\right|+\left|f^{\prime}(b)\right|\right. \\
& \quad+\frac{1}{2} \eta_{2}\left(\left|f^{\prime}(a)\right|,\left|f^{\prime}(b)\right|\right) \\
& \left.\quad+\frac{1}{2} \eta_{2}\left(\left|f^{\prime}(b)\right|,\left|f^{\prime}(a)\right|\right)\right\} .
\end{aligned}
$$

Furthermore, if we set $\eta_{2}(x, y)=x-y$ for all $x, y \in f(I)$, then we recapture Theorem 2 in [13].

The following result has been obtained in [14].

Lemma 19. Let $I \subseteq \mathbb{R}$ be an open invex set with respect to $\eta_{1}: I \times I \longrightarrow \mathbb{R}$. Also, suppose that $f: I \longrightarrow \mathbb{R}$ is a differentiable function. For any $a, b \in I$ with $\eta_{1}(b, a)>0$, if $f \in$ $L^{1}\left[a, a+\eta_{1}(b, a)\right]$, then the following equality for fractional integral holds:

$$
\begin{aligned}
& \frac{f(a)+f\left(a+\eta_{1}(b, a)\right)}{2} \\
& -\frac{\Gamma(\alpha+1)}{2 \eta_{1}^{\alpha}(b, a)}\left[J_{a^{+}}^{\alpha} f\left(a+\eta_{1}(b, a)\right)+J_{\left(a+\eta_{1}(b, a)\right)^{-}}^{\alpha} f(a)\right] \\
& =\frac{\eta_{1}(b, a)}{2} \int_{0}^{1}\left[t^{\alpha}-(1-t)^{\alpha}\right] f^{\prime}\left(a+t \eta_{1}(b, a)\right) d t
\end{aligned}
$$

Now we give the trapezoid-type inequality related to (31).

Theorem 20. Let $I \subseteq \mathbb{R}$ be an open invex set with respect to $\eta_{1}: I \times I \longrightarrow \mathbb{R}$ and let $f: I \longrightarrow \mathbb{R}$ be a differentiable function. Suppose that $\left|f^{\prime}\right|$ is an $\left(\eta_{1}, \eta_{2}\right)$-convex function on $I$ and, 
for any $a, b \in I$ with $\eta_{1}(b, a)>0, f^{\prime} \in L^{1}\left[a, a+\eta_{1}(b, a)\right]$. Then the following inequality for fractional integrals holds:

$$
\begin{aligned}
& \mid \frac{f(a)+f\left(a+\eta_{1}(b, a)\right)}{2} \\
& \quad-\frac{\Gamma(\alpha+1)}{2 \eta_{1}^{\alpha}(b, a)}\left[J_{a^{+}}^{\alpha} f\left(a+\eta_{1}(b, a)\right)\right. \\
& \left.\quad+J_{\left(a+\eta_{1}(b, a)\right)^{-}}^{\alpha} f(a)\right] \mid \leq \frac{\eta_{1}(b, a)}{2(\alpha+1)}\left(1-\frac{1}{2^{\alpha}}\right) \\
& \cdot\left[2\left|f^{\prime}(a)\right|+\eta_{2}\left(\left|f^{\prime}(b)\right|,\left|f^{\prime}(a)\right|\right)\right] .
\end{aligned}
$$

Proof. Using Lemma 19 and $\left(\eta_{1}, \eta_{2}\right)$-convexity of $\left|f^{\prime}\right|$, we get

$$
\begin{aligned}
& \mid \frac{f(a)+f\left(a+\eta_{1}(b, a)\right)}{2} \\
& \quad-\frac{\Gamma(\alpha+1)}{2 \eta_{1}^{\alpha}(b, a)}\left[J_{a^{+}}^{\alpha} f\left(a+\eta_{1}(b, a)\right)\right. \\
& \left.+J_{\left(a+\eta_{1}(b, a)\right)^{-}}^{\alpha} f(a)\right]\left|\leq \frac{\eta_{1}(b, a)}{2} \int_{0}^{1}\right| t^{\alpha}-(1-t)^{\alpha} \mid \\
& \cdot\left|f^{\prime}\left(a+t \eta_{1}(b, a)\right)\right| d t \leq \frac{\eta_{1}(b, a)}{2} \int_{0}^{1} \mid t^{\alpha} \\
& -(1-t)^{\alpha} \mid\left[\left|f^{\prime}(a)\right|+t \eta_{2}\left(\left|f^{\prime}(b)\right|,\left|f^{\prime}(a)\right|\right)\right] d t \\
& =\frac{\eta_{1}(b, a)}{2}\left\{\int_{0}^{1 / 2}\left[(1-t)^{\alpha}-t^{\alpha}\right]\right. \\
& \cdot\left[\left|f^{\prime}(a)\right|+t \eta_{2}\left(\left|f^{\prime}(b)\right|,\left|f^{\prime}(a)\right|\right)\right] d t \\
& +\int_{1 / 2}^{1}\left[t^{\alpha}-(1-t)^{\alpha}\right] \\
& . \\
& \left.\quad\left[\left|f^{\prime}(a)\right|+t \eta_{2}\left(\left|f^{\prime}(b)\right|,\left|f^{\prime}(a)\right|\right)\right] d t\right\} \\
& \quad=\frac{\eta_{1}(b, a)}{2(\alpha+1)}\left(1-\frac{1}{2^{\alpha}}\right)\left[2\left|f^{\prime}(a)\right|\right. \\
& \left.+\eta_{2}\left(\left|f^{\prime}(b)\right|,\left|f^{\prime}(a)\right|\right)\right],
\end{aligned}
$$

where the last equality can be obtained after some calculations in corresponding integrals and utilizing them.

Corollary 21. If in Theorem 20 we consider

(i) $\eta_{1}(x, y)=x-y$ for all $x, y \in I$, then we obtain

$$
\begin{aligned}
& \left|\frac{f(a)+f(b)}{2}-\frac{\Gamma(\alpha+1)}{2(b-a)^{\alpha}}\left[J_{a^{+}}^{\alpha} f(b)+J_{b^{-}}^{\alpha} f(a)\right]\right| \\
& \leq \frac{b-a}{2(\alpha+1)}\left(1-\frac{1}{2^{\alpha}}\right) \\
& \cdot\left[2\left|f^{\prime}(a)\right|+\eta_{2}\left(\left|f^{\prime}(b)\right|,\left|f^{\prime}(a)\right|\right)\right] .
\end{aligned}
$$

(ii) $\eta_{2}(x, y)=x-y$ for all $x, y \in f(I)$, then we have Theorem 2.5 in [14]. (iii) conditions of (i) and (ii) together, then we recapture Theorem 3 in [12].

As the last result by using Theorem 2 in [15], we obtain a refinement of Hermite-Hadamard inequality in connection with fractional integrals related to the increasing $\left(\eta_{1}, \eta_{2}\right)$ convex functions.

Theorem 22 (see [15]). If $f_{1}$ and $f_{2}$ are positive increasing functions on $[0,1]$, then

$$
\int_{0}^{1} f_{1}(x) d x \int_{0}^{1} f_{2}(x) d x \leq \int_{0}^{1} f_{1}(x) f_{2}(x) d x .
$$

Also if $f_{1}$ and $f_{2}$ are positive decreasing functions on $[0,1]$ and $K$ is an upper bound for $f_{1}$ and $f_{2}$, then $K-f_{1}$ and $K-f_{2}$ are positive increasing functions and we have

$$
\begin{gathered}
\int_{0}^{1}\left(K-f_{1}(x)\right) d x \int_{0}^{1}\left(K-f_{2}(x)\right) d x \\
\quad \leq \int_{0}^{1}\left(K-f_{1}(x)\right)\left(K-f_{2}(x)\right) d x
\end{gathered}
$$

which gives again

$$
\int_{0}^{1} f_{1}(x) d x \int_{0}^{1} f_{2}(x) d x \leq \int_{0}^{1} f_{1}(x) f_{2}(x) d x .
$$

Theorem 23. Let $I \subseteq \mathbb{R}$ be an invex set with respect to $\eta_{1}$ : $I \times I \longrightarrow \mathbb{R}$. Also let $f: I \longrightarrow \mathbb{R}$ be an increasing positive $\left(\eta_{1}, \eta_{2}\right)$-convex function. If, for each $a, b \in I$ with $\eta_{1}(b, a)>0$, $f \in L^{1}\left[a, a+\eta_{1}(b, a)\right]$ and $\eta_{2}$ is integrable on $f(I) \times f(I)$, then, for $\alpha>1$, the following inequalities hold:

$$
\begin{aligned}
& f\left(\frac{2 a+\eta_{1}(b, a)}{2}\right) \leq \frac{1}{\eta_{1}(b, a)} \int_{a}^{a+\eta_{1}(b, a)} f(x) d x \\
& \leq \frac{\Gamma(\alpha+1)}{2 \eta_{1}^{\alpha}(b, a)}\left[J_{a^{+}}^{\alpha} f\left(a+\eta_{1}(b, a)\right)+J_{\left(a+\eta_{1}(b, a)\right)^{-}}^{\alpha} f(a)\right] \\
& \leq f(a)+\frac{\eta_{2}(f(b), f(a))}{2} .
\end{aligned}
$$

Proof. From $\left(\eta_{1}, \eta_{2}\right)$-convexity of $f$, we have,

$$
f\left(a+t \eta_{1}(b, a)\right) \leq f(a)+t \eta_{2}(f(b), f(a)),
$$

and

$$
\begin{aligned}
& f\left(a+(1-t) \eta_{1}(b, a)\right) \\
& \quad \leq f(a)+(1-t) \eta_{2}(f(b), f(a)) .
\end{aligned}
$$

By adding these inequalities, we get

$$
\begin{aligned}
& f\left(a+t \eta_{1}(b, a)\right)+f\left(a+(1-t) \eta_{1}(b, a)\right) \\
& \quad \leq 2 f(a)+\eta_{2}(f(b), f(a)) .
\end{aligned}
$$


Multiplying both sides of (59) by $t^{\alpha-1}$ and integrating the resulting inequality with respect to $t$ over $[0,1]$ and using Theorem 22, we obtain

$$
\begin{aligned}
& \int_{0}^{1} t^{\alpha-1} d t \cdot \int_{0}^{1} f\left(a+t \eta_{1}(b, a)\right) d t+\int_{0}^{1} t^{\alpha-1} d t \\
& \cdot \int_{0}^{1} f\left(a+(1-t) \eta_{1}(b, a)\right) d t \\
& \quad \leq \int_{0}^{1} t^{\alpha-1} f\left(a+t \eta_{1}(b, a)\right) d t \\
& \quad+\int_{0}^{1} t^{\alpha-1} f\left(a+(1-t) \eta_{1}(b, a)\right) d t \\
& \quad \leq\left[2 f(a)+\eta_{2}(f(b), f(a))\right] \int_{0}^{1} t^{\alpha-1} d t .
\end{aligned}
$$

Using the changes of variables $x=a+t \eta_{1}(b, a)$ and $x=a+$ $(1-t) \eta_{1}(b, a)$, respectively, in above integrals, we have

$$
\begin{aligned}
& \frac{1}{\eta_{1}(b, a)} \int_{0}^{1} t^{\alpha-1} d t\left[\int_{a}^{a+\eta_{1}(b, a)} f(x) d x\right. \\
& \left.+\int_{a}^{a+\eta_{1}(b, a)} f\left(2 a+\eta_{1}(b, a)-x\right) d x\right] \\
& \leq \frac{\Gamma(\alpha)}{\eta_{1}^{\alpha}(b, a)}\left[J_{a^{+}}^{\alpha} f\left(a+\eta_{1}(b, a)\right)+J_{\left(a+\eta_{1}(b, a)\right)^{-}}^{\alpha} f(a)\right] \\
& \leq\left[2 f(a)+\eta_{2}(f(b), f(a))\right] \int_{0}^{1} t^{\alpha-1} d t .
\end{aligned}
$$

Since

$$
\int_{0}^{1} t^{\alpha-1} d t=\frac{1}{\alpha}
$$

and

$$
\begin{aligned}
& \int_{a}^{a+\eta_{1}(b, a)} f(x) d x \\
& \quad=\int_{a}^{a+\eta_{1}(b, a)} f\left(2 a+\eta_{1}(b, a)-x\right) d x
\end{aligned}
$$

we get

$$
\begin{aligned}
& \frac{1}{\eta_{1}(b, a)} \int_{a}^{a+\eta_{1}(b, a)} f(x) d x \\
& \leq \frac{\Gamma(\alpha+1)}{2 \eta_{1}^{\alpha}(b, a)}\left[J_{a^{+}}^{\alpha} f\left(a+\eta_{1}(b, a)\right)+J_{\left(a+\eta_{1}(b, a)\right)^{-}}^{\alpha} f(a)\right] \\
& \leq f(a)+\frac{\eta_{2}(f(b), f(a))}{2} .
\end{aligned}
$$

Furthermore, since $f$ is $\left(\eta_{1}, \eta_{2}\right)$-convex, then

$$
f\left(\frac{2 a+\eta_{1}(b, a)}{2}\right) \leq \frac{1}{\eta_{1}(b, a)} \int_{a}^{a+\eta_{1}(b, a)} f(x) d x
$$

which completes the proof.
Corollary 24. Let $f:[a, b] \longrightarrow \mathbb{R}$ be an increasing positive convex function. Then, for $\alpha>1$, the following inequalities hold:

$$
\begin{aligned}
f\left(\frac{a+b}{2}\right) & \leq \frac{1}{b-a} \int_{a}^{b} f(x) d x \\
& \leq \frac{\Gamma(\alpha+1)}{2(b-a)^{\alpha}}\left[J_{a^{+}}^{\alpha} f(b)+J_{b^{-}}^{\alpha} f(a)\right] \\
& \leq \frac{f(a)+f(b)}{2} .
\end{aligned}
$$

\section{Conclusions}

The convexity of a function is the basis for many inequalities in mathematics. Note that, in new problems related to the convexity, generalized notions about convex functions are required to obtain applicable results. One of these generalizations is the notion of $\left(\eta_{1}, \eta_{2}\right)$-convex functions which can generalize many inequalities related to convex functions such as Hermite-Hadamard inequality, Fejér inequality, and trapezoid-type and mid-point-type inequalities.

\section{Data Availability}

No data were used to support this study.

\section{Conflicts of Interest}

The authors declare that they have no conflicts of interest.

\section{Acknowledgments}

M. De La Sen is very grateful to the Spanish Government for its support by the European Regional Development Fund (ERDF) through Grant DPI2015-64766-R and to UPV/EHU for its support by Grant PGC 17/33.

\section{References}

[1] L. Fejér, Über die Fourierreihen, II. Math. Naturwiss. Anz Ungar. Akad. Wiss, vol. 24, 1906.

[2] S. M. Aslani, M. R. Delavar, and S. M. Vaezpour, "Inequalities of Fejér type related to generalized convex functions with applications," International Journal of Analysis and Applications, vol. 16, no. 1, pp. 38-49, 2018.

[3] M. S. Aslani, R. M. Delavar, and S. M. Vaezpour, "HermiteHadamard type integral inequalities for generalized convex functions," Journal of Inequalities and Special Functions, vol. 8, no. 1, pp. 17-33, 2018.

[4] A. Ben-Israel and B. Mond, "What is invexity?" The Journal of the Australian Mathematical Society. Series B. Applied Mathematics, vol. 28, no. 1, pp. 1-9, 1986.

[5] M. A. Hanson and B. Mond, "Convex transformable programming problems and invexity," Journal of Information \& Optimization Sciences. A Journal Devoted to Advances in Information Sciences, Optimization Sciences and Related Aspects, vol. 8, no. 2, pp. 201-207, 1987. 
[6] V. Jeyakumar, "Strong and weak invexity in mathematical programming," European Journal of Operational Research, vol. 55, pp. 109-125, 1985.

[7] M. Eshaghi Gordji, M. Rostamian Delavar, and M. De La Sen, "On $\varphi$-convex functions," Journal of Mathematical Inequalities, vol. 10, no. 1, pp. 173-183, 2016.

[8] M. R. Delavar and S. S. Dragomir, “On $\eta$-Convexity," Journal of Inequalities and Applications, vol. 20, no. 1, pp. 203-216, 2017.

[9] M. Rostamian Delavar and M. De La Sen, "Some generalizations of Hermite-Hadamard type inequalities," SpringerPlus, vol. 5, no. 1, article no. 1661, 2016.

[10] R. Gorenflo and F. Mainardi, "Fractional calculus: integral and differential equations of fractional order," in Fractals and Fractional Calculus in Continuum Mechanics, Springer, Vienna, Austria, 1997.

[11] I. Iscan, "Hermite-Hadamard-Fejer type inequalities for convex functions via fractional integrals," Studia Universitatis BabeșBolyai Mathematica, vol. 60, no. 3, pp. 355-366, 2015.

[12] M. Z. Sarikaya, E. Set, H. Yaldiz, and N. Başak, "HermiteHadamard's inequalities for fractional integrals and related fractional inequalities," Mathematical and Computer Modelling, vol. 57, no. 9-10, pp. 2403-2407, 2013.

[13] M. Iqbal, M. I. Bhatti, and K. Nazeer, "Generalization of inequalities analogous to Hermite-HADamard inequality via fractional integrals," Bulletin of the Korean Mathematical Society, vol. 52, no. 3, pp. 707-716, 2015.

[14] I. Iscan, "Hermite-Hadamards inequalities for preinvex functions via fractional integrals and related fractional inequalities," American Journal of Mathematical Analysis, vol. 1, no. 3, pp. 3338, 2013.

[15] M. D. Rostamian and F. Sajadian, "Hermite-Hadamard type integral inequalities for log- $\eta$-convex functions," Mathematics and Computer Science, vol. 1, no. 4, pp. 86-92, 2016. 


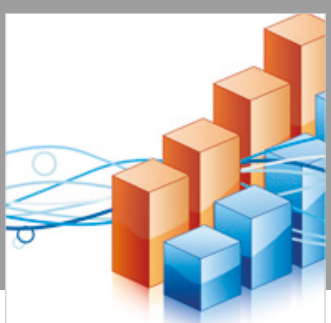

Advances in

Operations Research

\section{-n-m}
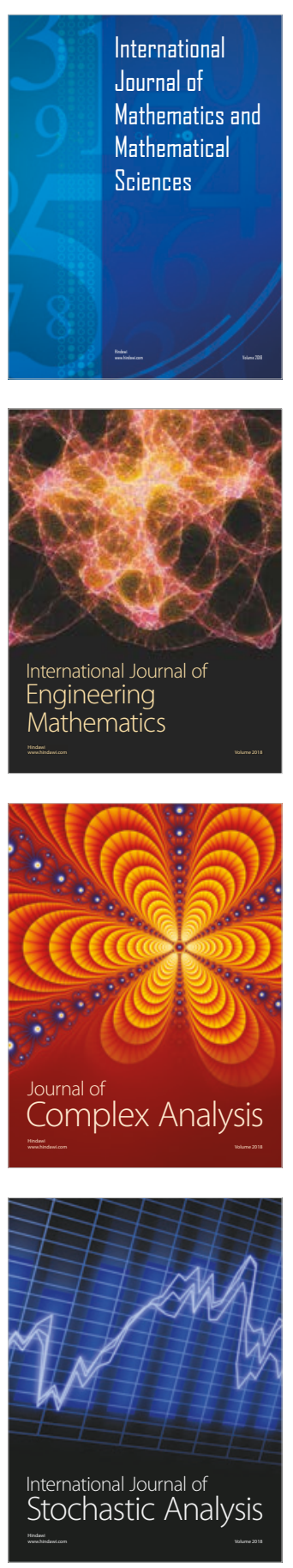
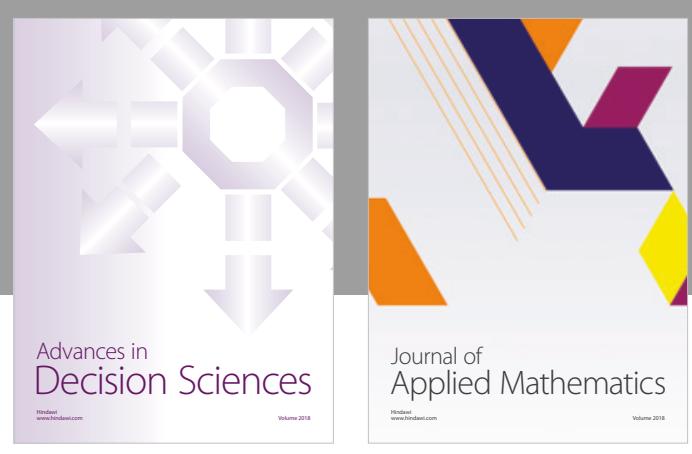

Journal of

Applied Mathematics
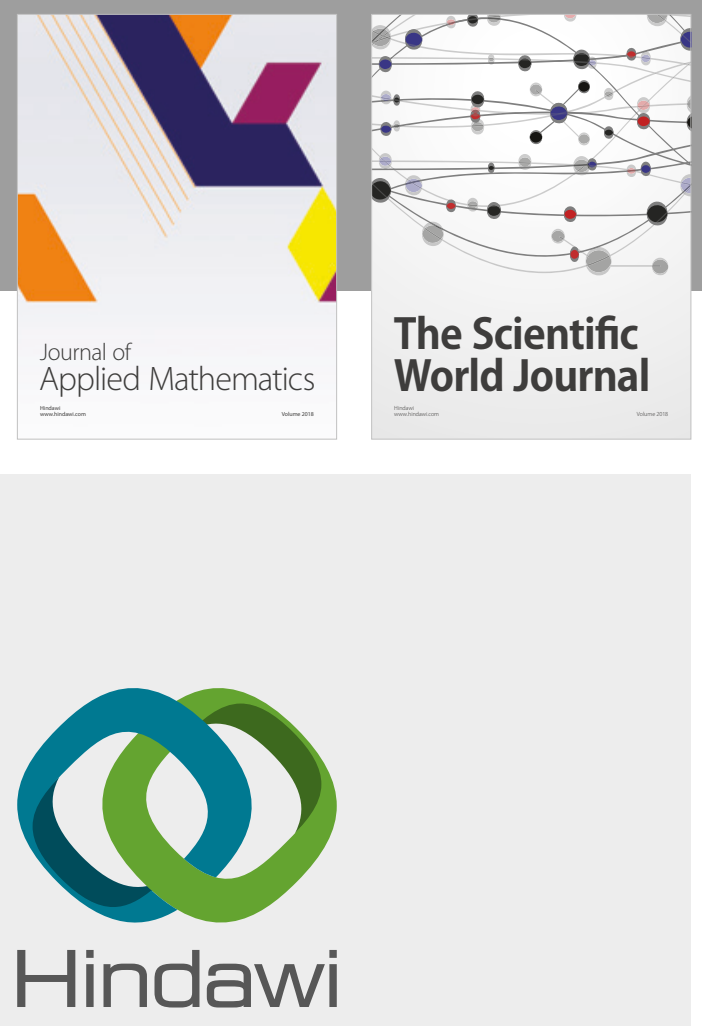

Submit your manuscripts at

www.hindawi.com

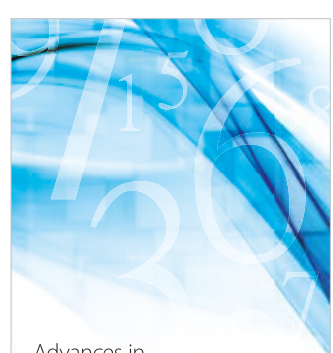

Advances in
Numerical Analysis
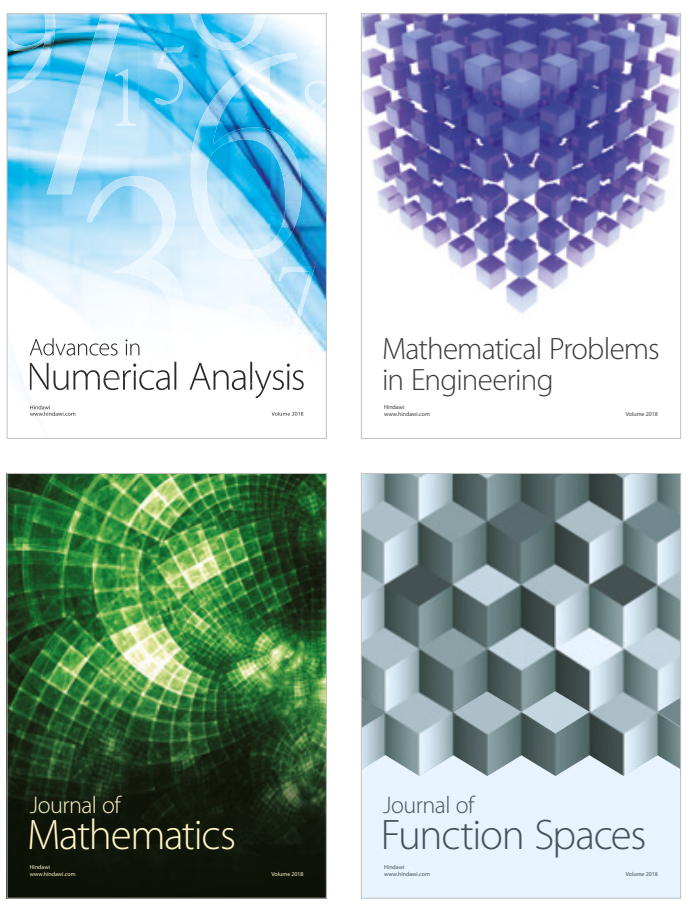

Mathematical Problems in Engineering

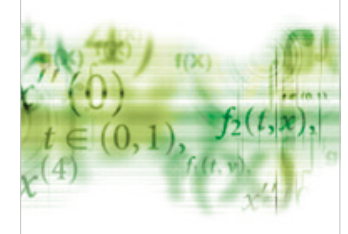

International Journal of

Differential Equations

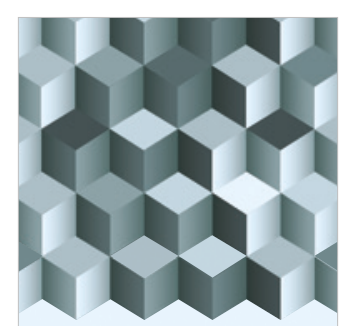

Journal of

Function Spaces

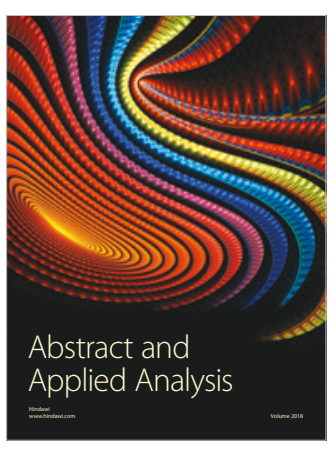

The Scientific

World Journal

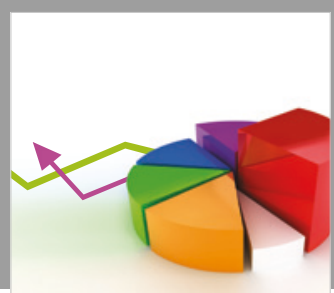

Journal of

Probability and Statistics
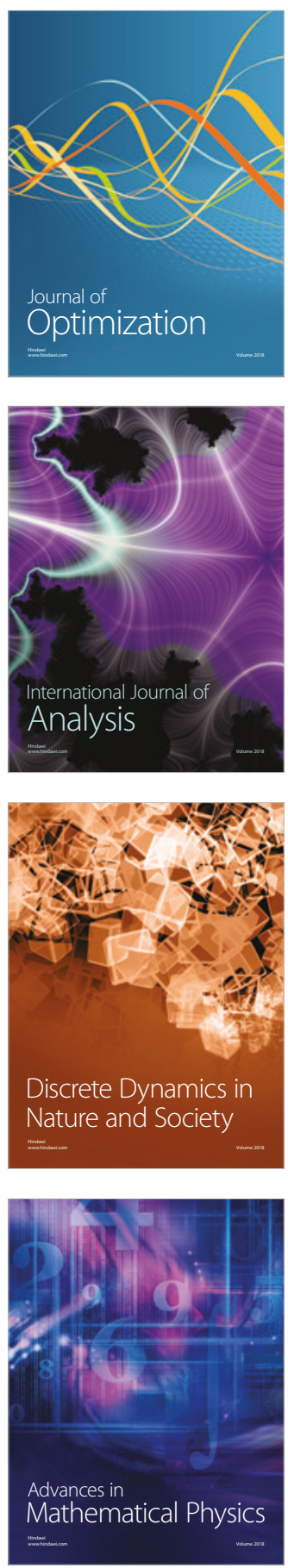